\title{
Photoacoustic imaging of human lymph nodes with endogenous lipid and hemoglobin contrast
}

James A. Guggenheim

Thomas J. Allen

Andrew Plumb

Edward Z. Zhang

Manuel Rodriguez-Justo

Shonit Punwani

Paul C. Beard

\section{SPIE.}




\section{Photoacoustic imaging of human lymph nodes with endogenous lipid and hemoglobin contrast}

\author{
James A. Guggenheim, ${ }^{a, \star}$ Thomas J. Allen, ${ }^{a}$ \\ Andrew Plumb, ${ }^{b}$ Edward Z. Zhang, ${ }^{a}$ \\ Manuel Rodriguez-Justo, ${ }^{c}$ Shonit Punwani, ${ }^{b}$ and \\ Paul C. Beard ${ }^{\mathrm{a}}$ \\ aUniversity College London, Department of Medical Physics \\ and Biomedical Engineering, Gower Street, London WC1E 6BT, \\ United Kingdom \\ bUniversity College London, Centre for Medical Imaging, 3rd Floor \\ East, 250 Euston Road, London NW1 2PG, United Kingdom \\ 'University College London, Cancer Institute, Department of \\ Research Pathology, Rockefeller Building, 21 University Street, \\ London WC1E 6JJ, United Kingdom
}

\begin{abstract}
Lymph nodes play a central role in metastatic cancer spread and are a key clinical assessment target. Abnormal node vascularization, morphology, and size may be indicative of disease but can be difficult to visualize with sufficient accuracy using existing clinical imaging modalities. To explore the potential utility of photoacoustic imaging for the assessment of lymph nodes, images of ex vivo samples were obtained at multiple wavelengths using a high-resolution three-dimensional photoacoustic scanner. These images showed that hemoglobin based contrast reveals nodal vasculature and lipid-based contrast reveals the exterior node size, shape, and boundary integrity. These two sources of complementary contrast may allow indirect observation of cancer, suggesting a future role for photoacoustic imaging as a tool for the clinical assessment of lymph nodes. (๑) 2015 Society of Photo-Optical Instrumentation Engineers (SPIE) [DOI: 10.1117/1.JBO.20.5.050504]
\end{abstract}

Keywords: photoacoustics; imaging; tomography; lymph node; cancer; lipid; hemoglobin; multiwavelength; metastasis.

Paper 150114LR received Feb. 26, 2015; accepted for publication Apr. 22, 2015; published online May 26, 2015.

\section{Introduction}

Lymph nodes are a foremost site of metastasis in many cancers, and establishing their level of involvement is important for disease staging, prognosis, and treatment planning. ${ }^{1,2}$ Extranodal spread-breaching of the lymph node boundary by cancerand neoangiogenesis can be indicators of cancer but are difficult to detect in vivo. ${ }^{2}$ Established clinical imaging modalities such as contrast-enhanced computed tomography and magnetic resonance imaging (MRI) can provide an estimate of the size of a lymph node. However, their inability to identify small cancerous deposits, reveal the detailed structure of the node, or visualize the internal vasculature limits diagnostic utility. ${ }^{3}$ Many clinical

*Address all correspondence to: James A. Guggenheim, E-mail: j.guggenheim@ucl.ac.uk situations, therefore, require histopathological assessment following biopsy, which is necessarily invasive and can lead to complications. $^{3}$

Photoacoustic imaging is an emerging modality ${ }^{4}$ that may be able to overcome some of the limitations of existing clinical imaging methods. Previous studies have demonstrated the potential to visualize cancer cells directly in excised human and animal nodes by exploiting the contrast provided by endogenous melanin $^{5}$ and an exogenous contrast agent ${ }^{6}$ respectively. Sentinel lymph nodes have also been imaged macroscopically in small animals with contrast agents flooding the node. ${ }^{7}$ While imaging cancer cells directly is clearly desirable, it is challenging in general because without exogenous contrast agents, most cancer cells (save for melanocytes) do not contain molecules that exhibit significant optical absorption.

In this study, a different approach, high-resolution photoacoustic imaging of human lymph nodes using intrinsic contrast based on hemoglobin $(\mathrm{Hb})$ and lipids, is explored. Contrast is correspondingly derived from internal vasculature and the fatty layer surrounding the node. This approach may allow indirect observation of cancer, for example by visualizing neoangiogenesis and revealing the distortion of a node boundary owing to internal tumor spreading.

\section{Materials and Methods}

Two formalin-fixed, macroscopically normal human mesenteric lymph nodes, "A" and "B," were imaged with a previously described photoacoustic imaging scanner, ${ }^{8}$ operating in tomography mode as shown in Fig. 1. The system comprises a widefield optical excitation source and an ultrasound detection system utilizing a planar Fabry-Perot polymer thin film sensor. The transduction mechanism of the sensor is one in which acoustically induced changes in its optical thickness produce a corresponding reflectivity modulation which is detected using a continuous-wave interrogation laser beam focused on the plane of the sensor. The interrogation beam is optically scanned point-by-point over the surface of the sensor in order to map the spatial distribution of the incident acoustic field in two dimensions. A three-dimensional (3-D) photoacoustic image is then reconstructed from these measurements using a fast k-space algorithm. ${ }^{8}$ The system can provide a lateral field-of-view (FOV) of $20 \mathrm{~mm} \times 20 \mathrm{~mm}$, a penetration depth in tissue of approximately $10 \mathrm{~mm}$, and a spatial resolution in the range 50 to $150 \mu \mathrm{m}$ depending on depth and lateral position. ${ }^{8}$

The lymph nodes were placed in acoustic contact with the sensor, submerged in either water or $1 \%$ intralipid solution, the latter creating a tissue-like scattering medium. The excitation source was a fiber-coupled, $30 \mathrm{~Hz}$, wavelength-tunable optical parametric oscillator (OPO) laser system (Spitlight 600, InnoLas Laser $\mathrm{GmbH}$, Krailling, Germany). The maximum fluence incident on the node surface was estimated to be $8 \mathrm{~mJ} / \mathrm{cm}^{2}$ per pulse. Although measurements were made in forward-mode for convenience, the transparent nature of the sensor allows convenient backward-mode operation as required for clinical use. ${ }^{8}$

Nodes were first imaged at excitation wavelengths of $530 \mathrm{~nm}$, where optical absorption by $\mathrm{Hb}$ is strong, ${ }^{9}$ and at $1210 \mathrm{~nm}$, at which there is a prominent lipid absorption peak. ${ }^{10}$ For convenient visualization, images of $\mathrm{Hb}$ and lipid were fused together by overlaying the $\mathrm{Hb}$ image on the lipid image and applying (to the $\mathrm{Hb}$ image) a level of opacity proportional to 


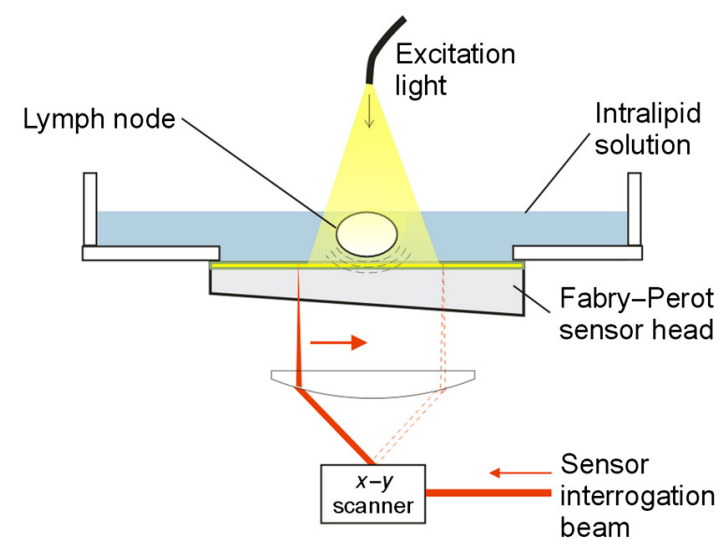

Fig. 1 Experimental setup for imaging lymph node samples.

the image intensity. Node A only was then imaged again at $1210 \mathrm{~nm}$ and at $920 \mathrm{~nm}$, a weaker lipid absorption peak, in order to compare the potential of imaging lipid structures at these two wavelengths. For comparison with the photoacoustic images, both nodes were also imaged using a clinical ultrasound system (ACUSON S2000, Siemens Healthcare, with a 18L6HD transducer) operated at $17 \mathrm{MHz}$, and a clinical 3T MRI system (Achieva, Philips Medical Systems) using the fat-sensitive component of an mDIXON sequence.

\section{Results and Discussion}

Figure 2 shows the photoacoustic images of both nodes acquired using excitation wavelengths of 530 and $1210 \mathrm{~nm}$. For node A, fly-through movies showing successive individual $x-y$ slices for each wavelength are available online and best illustrate the 3-D structure of the node. For both nodes, the $530 \mathrm{~nm}$ data show the blood vessels and the $1210 \mathrm{~nm}$ data show the fat surrounding the nodes. The vasculature within each of the nodes appears to be consistent with normal node physiology and there are apparent commonalities between the two nodes in terms of the size, structure, and distribution of the vessels As shown in Fig. 2, MRI is able to detect the node boundary based on fat contrast but with poor resolution and the ultrasound image reveals (with relatively low-resolution) the vessels as well as the fat. The photoacoustic images allow specific identification of the source of the contrast for both $\mathrm{Hb}$ and lipid owing to the spectral specificity of the technique.

The results show that it is possible to use photoacoustic imaging to provide intrinsically coregistered images of $\mathrm{Hb}$ and lipids in human lymph nodes and to interrogate the lipid with striking clarity. The presence of the node boundary in the images provides a useful spatial context for the blood vessel distribution and it is hypothesized that it may also allow identification of small changes in nodal structure due to malignant deposits.

While the lipid boundary was detectable at $1210 \mathrm{~nm}$ in these experiments, the in vivo penetration depth achievable at this wavelength is likely to be limited due to strong water absorption. Experiments were, therefore, also performed at $920 \mathrm{~nm}$, another lipid peak, at which both water and lipid absorption are significantly lower permitting greater penetration depth. Figure 3 shows the depth-resolved cross-sectional photoacoustic images of node A acquired in water using wavelengths of 920 and $1210 \mathrm{~nm}$. Also shown is a graph of the near-infrared (NIR) absorption spectra of water, lipids, and $\mathrm{Hb}^{9-11}$ The images show near identical features in the lipid around the node at both wavelengths. Although data are shown for selected slices only, the lipid is visible through the full depth of the node $(\sim 5 \mathrm{~mm})$ at both wavelengths making it possible to delineate the entire boundary.

The signal-to-noise ratio (SNR) at $920 \mathrm{~nm}$ is lower than at $1210 \mathrm{~nm}$, as expected due to the significantly higher absorption at $1210 \mathrm{~nm}$ and the modest penetration depths $(<6 \mathrm{~mm})$ involved in these experiments. At greater depths in vivo, it is anticipated that $920 \mathrm{~nm}$ will yield a higher SNR than $1210 \mathrm{~nm}$. Consider a tissue composed of $74 \%$ water, $1 \%$ blood, and $25 \%$ fat. Using typical values for the absorption coefficient, $\mu_{a}$, of these chromophores, ${ }^{9,10}$ and the reduced scattering coefficient,
Photo
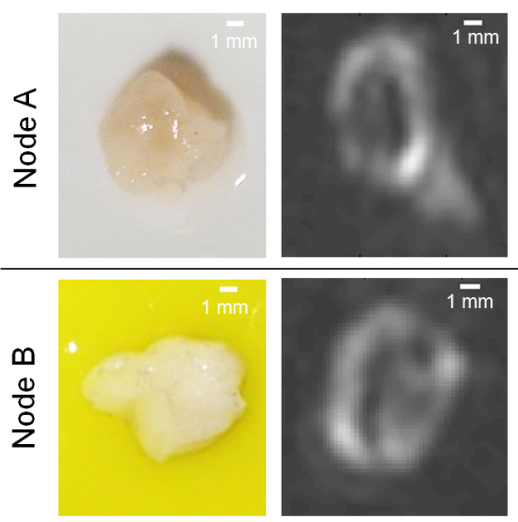

Ultrasound
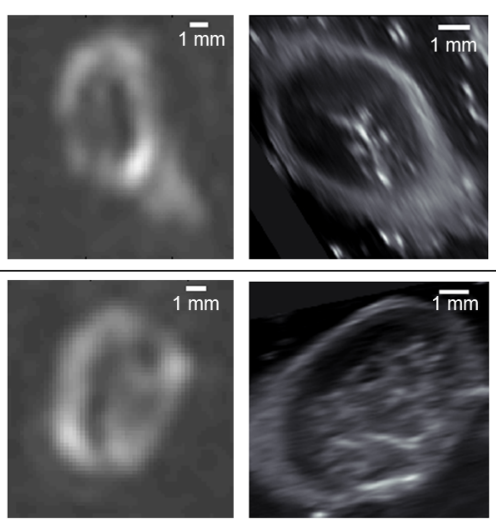

Photoacoustic Images (perpendicular MIPs)
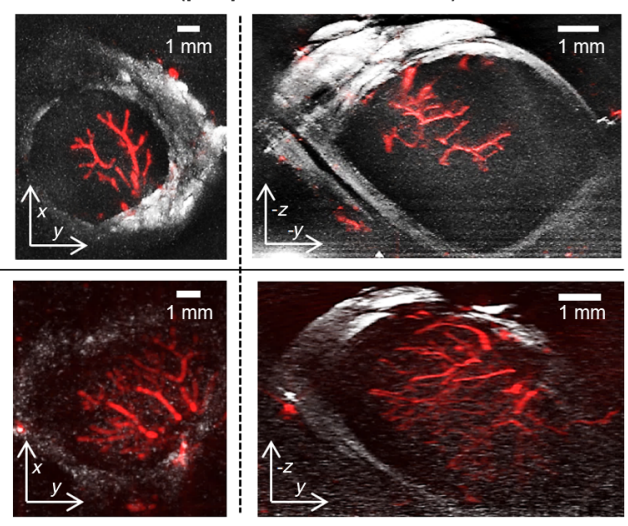

white $=1210 \mathrm{~nm}$, red $=530 \mathrm{~nm}$

Fig. 2 Images of two lymph node samples (node A and node B) acquired using macroscopic photography, clinical 3T magnetic resonance imaging (MRI), ultrasound (17 MHz), and photoacoustic imaging. The photoacoustic images are maximum intensity projections of the three-dimensional (3-D) image datasets obtained at $530 \mathrm{~nm}$ [red: hemoglobin $(\mathrm{Hb})]$ and $1210 \mathrm{~nm}$ (gray: lipid), fused together and visualized at two perpendicular orientations. The photoacoustic images of node $A$ were obtained with step sizes of 50 and $100 \mu \mathrm{m}$ for the lipid and $\mathrm{Hb}$ data, respectively, with a field-of-view (FOV) of $9 \times 11 \mathrm{~mm}$. A fly-through movie of node $A$ can be viewed online (supplementary information online: Video 1, MOV, $6768 \mathrm{~KB}$ ). The images of node $B$ were obtained with $200-\mu \mathrm{m}$ step sizes with an FOV of $10 \times 11 \mathrm{~mm}$. The sensor bandwidth $(-3 \mathrm{~dB})$ for both datasets was $31 \mathrm{MHz}$ [URL: http://dx.doi.org/10.1117/1.JBO.20.5.050504.1]. 
(a)
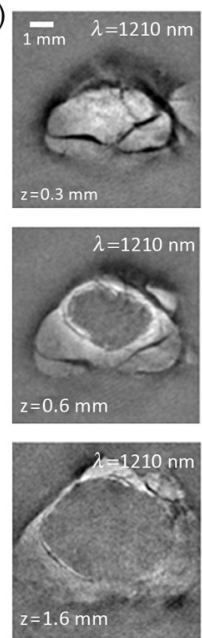
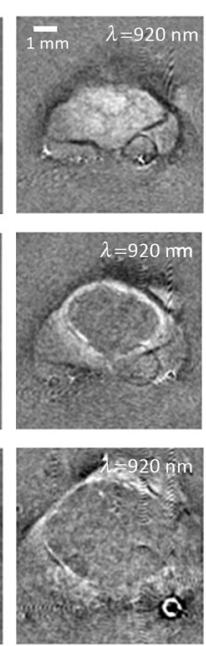
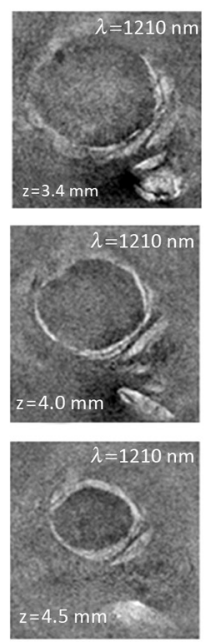
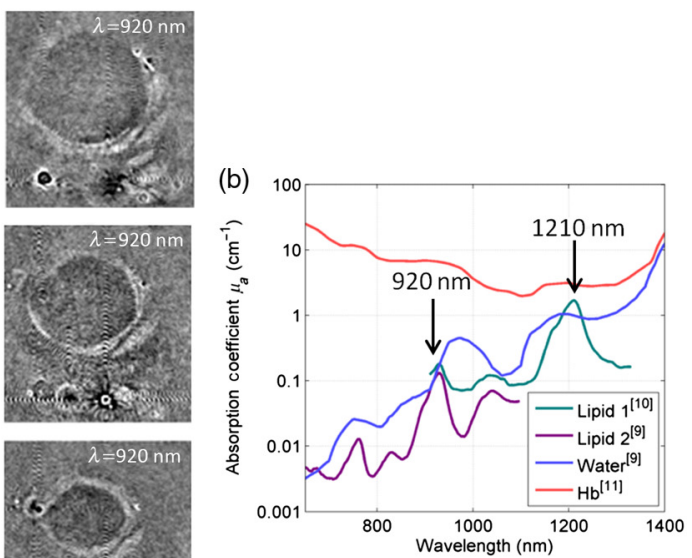

Fig. 3 (a) Photoacoustic images of node A obtained at 1210 and $920 \mathrm{~nm}$ and (b) near-infrared (NIR) absorption spectra of $\mathrm{Hb}$, water, and lipids. The images are cross-sectional slices at selected depths as indicated. The images were obtained with a step size of $100 \mu \mathrm{m}$, with an FOV of $9 \times 9 \mathrm{~mm}$. The sensor bandwidth $(-3 \mathrm{~dB})$ was $40 \mathrm{MHz}$.

$\mu_{s}^{\prime}$, of fatty tissue, ${ }^{9}$ the effective attenuation coefficient for the tissue can be calculated as $\mu_{\text {eff }}=\sqrt{3 \mu_{a}\left(\mu_{a}+\mu_{s}^{\prime}\right)}$. Transmittance at depth $d$ can then be estimated by $T(d)=e^{-\mu_{\text {eff }} d}$. Using values of 0.1 and $1.7 \mathrm{~cm}^{-1}$ for $\mu_{a}$ and 12.2 and $10.2 \mathrm{~cm}^{-1}$ for $\mu_{s}^{\prime}$, for 920 and $1210 \mathrm{~nm}$, respectively, $\mu_{\mathrm{eff}}$ is calculated to be 1.9 and $7.8 \mathrm{~cm}^{-1}$. Using this model, at $7 \mathrm{~mm}$ (approximate minimum lymph node depth), the transmittance at $920 \mathrm{~nm}$ is $17 \%$ and at $1210 \mathrm{~nm}$ it is $1.2 \%$. This corresponds approximately to the difference in absorption of lipids at the two wavelengths, therefore, the greater loss in light due to attenuation in the surrounding medium is compensated by the increased absorption attained at the higher wavelength and the predicted SNR at the two wavelengths is similar at this depth. Beyond this depth, it becomes exponentially more beneficial to use $920 \mathrm{~nm}$. For example, at a depth of $12.5 \mathrm{~mm}$, the calculated transmittance is $4.5 \%$ at $920 \mathrm{~nm}$ and $0.034 \%$ at $1210 \mathrm{~nm}$ which, correcting for the difference in absorption, leads to a predicted 10-fold improvement in SNR at $920 \mathrm{~nm}$.

\section{Conclusion}

Photoacoustic imaging has been used to obtain high-resolution 3-D images of the structure and internal vasculature of human lymph nodes based on $\mathrm{Hb}$ and lipid contrast. Compared with clinical ultrasound and MRI, the photoacoustic images exhibit high resolution and contrast as well as providing chromophore specificity. Lipid imaging was shown to be possible at 920 and $1210 \mathrm{~nm}$ excitation wavelengths. SNR at $920 \mathrm{~nm}$ was lower than that at $1210 \mathrm{~nm}$ as expected for the penetration depths relevant ( $<6 \mathrm{~mm}$ ) to this study. However, calculations suggest that, for greater depths, $920 \mathrm{~nm}$ may provide a higher SNR in vivo owing to weaker attenuation by background tissues. As well as being relevant to photoacoustic lymph node imaging, this finding may allow visualization of other lipid rich structures such as nerves at depths beyond that achievable using the more commonly used $1210 \mathrm{~nm}$ lipid excitation wavelength. ${ }^{12}$ It is concluded that photoacoustic imaging with endogenous contrast has the potential to assess cancer in the human lymph node by providing images of node vasculature and morphology. Further work will be undertaken to establish the clinical utility of the approach by imaging nodes with known malignant deposits and by carrying out in vivo studies.

\section{Acknowledgments}

The authors acknowledge support by King's College London and University College London Comprehensive Cancer Imaging Centre, Cancer Research UK \& Engineering and Physical Sciences Research Council, in association with the Medical Research Council and Department of Health, UK and European Union project FAMOS (FP7 ICT, Contract 317744).

\section{References}

1. J. A. Joyce and J. W. Pollard, "Microenvironmental regulation of metastasis," Nat. Rev. Cancer 9(4), 239-252 (2009).

2. R. B. J. de Bondt et al., "Detection of lymph node metastases in head and neck cancer: a meta-analysis comparing US, USgFNAC, CT and MR imaging," Eur. J. Radiol. 64(2), 266-272 (2007).

3. C. L. Bayer, P. P. Joshi, and S. Y. Emelianov, "Photoacoustic imaging: a potential tool to detect early indicators of metastasis," Expert Rev. Med. Devices 10(1), 125-134 (2013).

4. P. Beard, "Biomedical photoacoustic imaging," Interface Focus 1(4), 602-631 (2011).

5. J. Jose et al., "Initial results of imaging melanoma metastasis in resected human lymph nodes using photoacoustic computed tomography," J. Biomed. Opt. 16(9), 096021 (2011).

6. D. J. Grootendorst et al., "Evaluation of superparamagnetic iron oxide nanoparticles (Endorem $®$ ) as a photoacoustic contrast agent for intraoperative nodal staging," Contrast Media Mol. Imaging 8(1), 83-91 (2013).

7. K. H. Song et al., "Noninvasive photoacoustic identification of sentinel lymph nodes containing methylene blue in vivo in a rat model," J. Biomed. Opt. 13(5), 054033 (2008).

8. E. Zhang, J. Laufer, and P. Beard, "Backward-mode multiwavelength photoacoustic scanner using a planar Fabry-Perot polymer film ultrasound sensor for high-resolution three-dimensional imaging of biological tissues," Appl. Opt. 47(4), 561-577 (2008).

9. S. L. Jacques, "Optical properties of biological tissues: a review," Phys. Med. Biol. 58(14), 5007-5008 (2013).

10. C. Tsai, J. Chen, and W. Wang, "Near-infrared absorption property of biological soft tissue constituents," J. Med. Biol. Eng. 21(1), 7-13 (2001).

11. J. T. Kuenstner, K. H. Norris, and W. F. McCarthy, "Measurement of hemoglobin in unlysed blood by near-infrared spectroscopy," Appl. Spectrosc. 48(4), 484-488 (1994).

12. T. J. Allen et al., "Spectroscopic photoacoustic imaging of lipid-rich plaques in the human aorta in the 740 to $1400 \mathrm{~nm}$ wavelength range," J. Biomed. Opt. 17(6), 061209 (2012). 